\title{
A Multicolor Study of AGNs and BL Lacs
}

\author{
W.-H. Sun, Yu-Ya Su, and Hsiao-Feng Tang \\ Institute of Astronomy, National C'entral University, Chung-Li, Taiwan \\ $320, R O C$
}

\author{
Matthew A. Malkan \\ Department of Astronomy, UCLA, Los Angeles, CA 90024, USA
}

\author{
Remington P.S. Stone \\ Lick Observatory, Mt. Hamilton, CA 95064, USA
}

\begin{abstract}
We present preliminary results of two monitoring projects on AGNs and BL Lacs with four intermediate-band optical filters at Lick Observatory, South African Astronomical Observatory, and Siding Spring Observatory. We applied differential photometry between the target and non-variable field stars to derive the variability amplitudes. All the AGNs varied in all wavebands, with amplitudes from $30 \%$ to $90 \%$. The variations in shorter wavelength filters are always larger than in longer wavelength filters. We did not observe significant continuum slope changes. Continuum variations of BL Lacs up to $0.2 \mathrm{mag}$ in 20 minutes were observed. Interestingly, we found a time lag of 6-8 minutes between two seemingly correlated variations observed in the intermediate $U$ band with the 1-m telescope and in broad $V$ band with the 24-in. telescope at SSO.
\end{abstract}

\section{Introduction and Observations}

We measured the variability of several AGNs (at Lick) and BL Lacs (at SAAO) at $3890 \AA(U), 5750 \AA(V), 8020 \AA(I)$, and $9750 \AA(Z)$ with two-inch square intermediate-band filters via differential photometry, to study the energy-transfer mechanisms in the putative black-hole accretion disks. We used only $U, V$, and broad-band $V$ in the Australia run. These filters are derived from the BATC (Beijing-Arizona-Taipei-Connecticut) Sky Survey program currently being carried out by the US, the Mainland, and Taiwan, at the Schmidt telescope of Beijing Astronomical Observatory (BAO). Typical bandwidths of these filters are about $250-300 \AA$, significantly narrower than traditional broad-band filters (bandwidth roughly $1000-1500 \AA$ ), and thus allow us to narrow down the emission regions on the putative accretion disk.

The reason that we prefer differential photometry to low-resolution spectrophotometry is that the former will give an accuracy of $1 \%$ or better while the latter usually gives an accuracy of $5-10 \%$, which is not sufficient to detect small-scale variations. 
At the end of the AGN monitoring program, we selected five AGNs from the sample which have more complete data for analysis. The five objects are Mkn 335, Mkn 509, Akn 120, MCG 8-11-11, and NGC 4151. From the BL Lac program, we selected PKS $0521-365$ for analysis since this object exhibits short-term, large-amplitude variability over the monitoring period.

Aperture photometry was performed to obtain the instrumental magnitudes of the target AGNs as well as several field stars. The scientific goal can be achieved with instrumental magnitudes since we are looking only for variations. Typically 8-10 field stars were examined, and about three field stars were inter-compared with their differences among themselves over the 1.5-year monitoring period. This process allows the detection of any variations in these stars, and help to identify the non-variable field stars which can be used in the comparison with AGNs. The typical differences between field stars for Mkn 335 are $0.064 \mathrm{mag}$ for $U, 0.022 \mathrm{mag}$ for $V, 0.024 \mathrm{mag}$ for $I$, and $0.023 \mathrm{mag}$ for $Z$.

We applied the same reduction and differential photometry technique to the $\mathrm{BL}$ Lac fields in the SAAO run and the ANU run.

\section{Discussion}

We find significant variations $(30 \%$ to $90 \%)$ in the four selected wavebands of the target $\mathrm{AGN}$ in the monitoring program. It is found that the variability amplitude at shorter wavelengths is greater than at longer wavelengths, regardless of whether the AGN is getting brighter or fainter.

From the light curves of the five AGNs, it is not obvious there are any time delays. We applied cross-correlation analysis to the light curves in neighboring wavebands and determined that any time delays should be less than 15 days, the minimum temporal resolution that our 30-day interval could provide. In addition, we did not observe significant slope change.

For the BL Lac objects, we observed very short-term variability in both the $\mathrm{SAAO}$ and the ANU runs. In the SAAO run, we saw a change of $0.1 \mathrm{mag}$ in half an hour. In the $A N U$ run, however, the variability became more extreme. A variation of $0.2 \mathrm{mag}$ in less than 20 minutes was observed. Also, we found an apparent time-delay between the observations in $U$ (intermediate band) and in $V$ (broad band). This time delay provides a stringent constraint to the modeling of the emission mechanisms. Further observations to confirm this observation are in progress.

Acknowledgments. This research program is supported by NSC through grant number NSC 84-2112-M-008-018. 